\title{
Article \\ Preserving the Shape of Functions by Applying Multidimensional Schoenberg-Type Operators
}

\author{
Camelia Liliana Moldovan ${ }^{+}(\mathbb{D})$ and Radu Păltănea *,+ ${ }^{*}$ \\ Faculty of Mathematics and Computer Science, Transilvania University of Brasov, 500036 Brasov, Romania; \\ moldovancamelia.liliana@unitbv.ro \\ * Correspondence: radu.paltanea@unitbv.ro \\ + These authors contributed equally to this work.
}

check for

updates

Citation: Moldovan, C.L.; Păltănea,

R. Preserving the Shape of Functions by Applying Multidimensional Schoenberg-Type Operators. Symmetry 2021, 13, 1016. https:/ /doi.org/10.3390/sym13061016

Academic Editor: Ioan Rașa

Received: 30 April 2021

Accepted: 2 June 2021

Published: 5 June 2021

Publisher's Note: MDPI stays neutral with regard to jurisdictional claims in published maps and institutional affiliations.

Copyright: (c) 2021 by the authors. Licensee MDPI, Basel, Switzerland. This article is an open access article distributed under the terms and conditions of the Creative Commons Attribution (CC BY) license (https:// creativecommons.org/licenses/by/ $4.0 /)$.
Abstract: The paper presents a multidimensional generalization of the Schoenberg operators of higher order. The new operators are powerful tools that can be used for approximation processes in many fields of applied sciences. The construction of these operators uses a symmetry regarding the domain of definition. The degree of approximation by sequences of such operators is given in terms of the first and the second order moduli of continuity. Extending certain results obtained by Marsden in the one-dimensional case, the property of preservation of monotonicity and convexity is proved.

Keywords: multidimensional splines; multidimensional Schoenberg-type operators; order of approximation; monotonicity; convexity

\section{Introduction}

The theory of splines approximation was founded by Schoenberg and became one of the main chapters of approximation theory. Now there is a vast literature dedicated to spline approximation. We refer the reader to the monograph of Schumaker [1] for historical notes. The success of this type of approximation is due both to the nice mathematical theory and to the great efficiency in practical applications. In practice, the spline approximation is more efficient then the polynomial approximation.

In [2], Schoenberg considered also a particular method of approximation of functions by splines, with the aid of certain positive linear operators, which are named the Schoenberg operators. Important contributions in the study of these operators are due to Marsden [3].

In more recent times, the topic of one-dimensional Schoenberg spline operators are presented in papers written for instance in Gonska [4-7], Tachev [4-8], Beutel [5-7,9], Kacsó [4-7] and our papers [10-12].

The subject of multidimensional spline is developed in many papers. We can specify here the paper [13] where the approximation of functions using multivariate splines is presented and the monograph [14], which is dedicated to the theory of multivariate splines. We mention also the paper [15] where the multivariate polynomial interpolation is approached, the paper [16] where a computationally effective way to construct stable bases on general non-degenerate lattices is presented, Reference [17] where the subject of Hermite-vector splines and multi-wavelets is developed and the paper [18] in which a generalization of bases, namely B-spline frames, is approached. Estimates of approximation by linear operators in the multidimensional case are established in [19].

As exemplification of the application of the Schoenberg operators in practice we mention the recent paper [20] where one-dimensional Schoenberg spline operators were used, obtaining a substantial improvement of the clear sky models which estimate the direct solar irradiance.

The present paper is a continuation of paper [12], where two-dimensional Schoenberg operators were considered. Now we extend this definition in multidimensional case and we establish certain properties of them. 
Several important connections with symmetry exist in this study. Because these operators present a symmetry in their construction, the computation of their moments is made by symmetry. The symmetry is also used in establishing the estimates with second order moduli, which are defined with the aid of finite symmetric differences. On the other hand, we study the property of preservation of convexity and this property can be described using the Hessian of functions, which is a symmetric quadratic form.

\section{Multidimensional Schoenberg-Type Operators on Arbitrary Nodes}

We consider the integers $j, m, 1 \leq j \leq m ; n_{j}>0 ; k_{j}>0$; the vector $\left(x_{1}, \ldots, x_{m}\right) \in$ $[0,1]^{m}$ and the knots sequences $\Delta_{n_{j}, k_{j}}$

$0=v_{-k_{j}}=v_{-k_{j}+1}=\ldots=v_{0_{j}}<v_{1_{j}}<v_{2_{j}}<\ldots<v_{n_{j}}=v_{n_{j}+1}=\ldots=v_{n_{j}+k_{j}}=1$.

The Greville abscissas associated with division $\Delta_{n_{j}, k_{j}}, 1 \leq j \leq m$ have the next form

$$
\xi_{i_{j}}:=\frac{v_{i_{j}+1}+v_{i_{j}+2}+\ldots+v_{i_{j}+k_{j}}}{k_{j}}, \quad-k_{j} \leq i_{j} \leq n_{j}-1 .
$$

The B-splines $N_{i_{j}, k_{j}}\left(x_{j}\right), 1 \leq j \leq m$ depending on $\Delta_{n_{j}, k_{j}}$ are

$$
N_{i_{j}, k_{j}}\left(x_{j}\right)=\left(v_{i_{j}+k_{j}+1}-v_{i_{j}}\right)\left[v_{i_{j}}, v_{i_{j}+1}, \ldots, v_{i_{j}+k_{j}+1}\right]\left(\cdot-x_{j}\right)_{+}^{k_{j}},-k_{j} \leq i_{j} \leq n_{j}-1 .
$$

When $x_{j} \in\left[v_{q_{j}}, v_{q_{j}+1}\right]$ with $0 \leq q_{j} \leq n_{j}-1,1 \leq j \leq m$ we have:

$N_{i_{j}, k_{j}}\left(x_{j}\right)=0$, for $i_{j}<q_{j}-k_{j}$ or $i_{j} \geq q_{j}+1$, and $N_{i_{j}, k_{j}}\left(x_{j}\right) \geq 0$, for $q_{j}-k_{j} \leq i_{j} \leq q_{j}$.

The next relations take place

$$
\sum_{i_{j}=-k_{j}}^{n_{j}-1} N_{i_{j}, k_{j}}\left(x_{j}\right)=1, \text { for } x_{j} \in[0,1]
$$

and

$$
\sum_{i_{j}=-k_{j}}^{n_{j}-1} \xi_{i_{j}} N_{i_{j}, k_{j}}\left(x_{j}\right)=x_{j} \text { for } x_{j} \in[0,1],
$$

where $1 \leq j \leq m$.

We consider $\Delta^{\star}=\Delta_{n_{1}, k_{1}} \times \Delta_{n_{2}, k_{2}} \times \ldots \times \Delta_{n_{m}, k_{m}}$

$$
\Delta^{\star}:=\left\{\left(v_{i_{1}}, \ldots, v_{i_{m}}\right),-k_{j} \leq i_{j} \leq n_{j}+k_{j}, 1 \leq j \leq m\right\} .
$$

Definition 1. Multidimensional Schoenberg-type operator associated with $\Delta^{\star}$ has the form

$$
\begin{aligned}
& \left(S_{\Delta^{\star}} f\right)\left(x_{1}, \ldots, x_{m}\right) \\
= & \sum_{i_{1}=-k_{1}}^{n_{1}-1} \ldots \sum_{i_{m}=-k_{m}}^{n_{m}-1}\left(N_{i_{1}, k_{1}} \otimes \ldots \otimes N_{i_{m}, k_{m}}\right)\left(x_{1}, \ldots, x_{m}\right) f\left(\xi_{i_{1}}, \ldots, \xi_{i_{m}}\right),
\end{aligned}
$$

where $f:[0,1]^{m} \rightarrow \mathbb{R}$, and $x=\left(x_{1}, \ldots, x_{m}\right) \in[0,1]^{m}$.

\section{Remark 1.}

(i) Symmetrizing the knots $v_{i_{i}}$ on each components by function $\sigma(x)=1-x, x \in[0,1]$ one obtains also a Schoenberg-type operators of the same degree. If the knots are equidistant, one obtains the same Schoenberg-type operators. 
(ii) For $x_{j} \in\left[v_{q_{j}}, v_{q_{j}+1}\right]$, with $0 \leq q_{j} \leq n_{j}-1$ and $1 \leq j \leq m$, then

$$
\begin{aligned}
& \left(S_{\Delta^{\star}} f\right)\left(x_{1}, \ldots, x_{m}\right) \\
= & \sum_{i_{1}=q_{1}-k_{1}}^{q_{1}} \ldots \sum_{i_{m}=q_{m}-k_{m}}^{q_{m}} N_{i_{1}, k_{1}}\left(x_{1}\right) \ldots N_{i_{m}, k_{m}}\left(x_{m}\right) f\left(\xi_{i_{1}}, \ldots, \xi_{i_{m}}\right) .
\end{aligned}
$$

(iii) Multidimensional Schoenberg-type operators are linear and positive.

(iv) $S_{\Delta^{\star}}$ is a polynomial of degree at most $k_{j}$ in each variable $x_{j}, 1 \leq j \leq m$, on each domain $\left[v_{i_{1}-1}, v_{i_{1}}\right] \times \ldots \times\left[v_{i_{m}-1}, v_{i_{m}}\right]$, with $0 \leq i_{j} \leq n_{j}-1$ and $1 \leq j \leq m$.

(v) $S_{\Delta^{\star}}$ is a B-spline in each variable.

(vi) Multidimensional Schoenberg-type operators admit partial continuous derivatives on $[0,1]^{m}$, since

$$
\begin{aligned}
& \left(\frac{\partial^{i_{1}+\ldots+i_{m}}}{\partial x_{1}^{i_{1}} \ldots \partial x_{m}^{i_{m}}}\left(S_{\Delta^{\star}} f\right)\right)\left(x_{1}, \ldots, x_{m}\right) \\
= & \sum_{i_{1}=-k_{1}}^{n_{1}-1} \frac{\partial^{i_{1}}}{\partial x_{1}^{i_{1}}} N_{i_{1}, k_{1}}\left(x_{1}\right) \ldots \sum_{i_{m}=-k_{m}}^{n_{m}-1} \frac{\partial^{i_{m}}}{\partial x_{m}^{i_{m}}} N_{i_{m}, k_{m}}\left(x_{m}\right) f\left(\xi_{i_{1}}, \ldots, \xi_{i_{m}}\right),
\end{aligned}
$$

where $0 \leq i_{j} \leq k_{j}, 1 \leq j \leq m$.

We consider the next functions: $e_{0} \in C\left([0,1]^{m}\right), e_{0}\left(x_{1}, \ldots, x_{m}\right)=1$ and $\pi_{j} \in C\left([0,1]^{m}\right)$, $\pi_{j}\left(x_{1}, \ldots, x_{m}\right)=x_{j}$, for $\left(x_{1}, \ldots, x_{m}\right) \in[0,1]^{m}, 1 \leq j \leq m$.

Proposition 1. For $\left(x_{1}, \ldots, x_{m}\right) \in[0,1]^{m}$ we have

(i) $\left(S_{\Delta^{\star}} e_{0}\right)\left(x_{1}, \ldots, x_{m}\right)=1$;

(ii) $\left(S_{\Delta^{\star}} \pi_{j}\right)\left(x_{1}, \ldots, x_{m}\right)=x_{j}, 1 \leq j \leq m$;

(iii)

$$
S_{\Delta^{\star}}\left(\prod_{j=1}^{m} \pi_{j}\right)\left(x_{1}, \ldots, x_{m}\right)=\prod_{j=1}^{m} x_{j}
$$

We use the next notations: $e_{1}(t)=t, t \in[0,1] ; e_{0}$ for the constant function equal to 1 , on $[0,1]^{m}, 1 \leq j \leq m$ and $\Delta_{j}, 1 \leq j \leq m$ denotes the knot sequence use to one-dimensional Schoenberg operators.

Proposition 2. For $\left(x_{1}, \ldots, x_{m}\right) \in[0,1]^{m}$ we have

(i) $\left(S_{\Delta^{\star}}\left(\pi_{j}-x_{j} e_{0}\right)\right)\left(x_{1}, \ldots, x_{m}\right)=0$,

(ii)

$$
\left(S_{\Delta^{\star}}\left(\prod_{j=1}^{m}\left(\pi_{j}-x_{j} e_{0}\right)\right)\right)\left(x_{1}, \ldots, x_{m}\right)=0,
$$

(iii) $\left(S_{\Delta^{\star}}\left(\pi_{j}-x_{j} e_{0}\right)^{m}\right)\left(x_{1}, \ldots, x_{m}\right)=\left(S_{\Delta_{j}}\left(e_{1}-x_{j} e_{0}\right)^{m}\right)\left(x_{j}\right), 1 \leq j \leq m$.

Theorem 1. For multidimensional Schoenberg-type operators

$$
\left(S_{\Delta^{\star}} f\right)\left(x_{1}, \ldots, x_{m}\right)=\sum_{i_{1}=-k_{1}}^{n_{1}-1} \ldots \sum_{i_{m}=-k_{m}}^{n_{m}-1} N_{i_{1}, k_{1}}\left(x_{1}\right) \ldots N_{i_{m}, k_{m}}\left(x_{m}\right) f\left(\xi_{i_{1}}, \ldots, \xi_{i_{m}}\right),
$$


to converge uniformly on $[0,1]^{m}$ to continuous function $f$, it is sufficient that for any $\eta>0$

$$
\sum_{\substack{-k_{j} \leq i_{j} \leq n_{j}-1,1 \leq j \leq m \\\left\|\left(\xi_{i_{1}}, \ldots, \xi_{i m}\right)-\left(x_{1}, \ldots, x_{m}\right)\right\| \geq \eta}} N_{i_{1}, k_{1}}\left(x_{1}\right) \ldots N_{i_{m}, k_{m}}\left(x_{m}\right) \rightarrow 0,
$$

uniformly for $0 \leq x_{j} \leq 1$, when $n_{j} \rightarrow \infty, 1 \leq j \leq m$.

Proof. We consider (10) is fulfilled.

From $f$ continue function on $[0,1]^{m}$, we have $\forall \varepsilon>0, \exists \eta_{\varepsilon}>0$ such that for any $\left(x_{i_{1}}, \ldots, x_{i_{m}}\right) \in[0,1]^{m}$ and $\left(x_{i_{1}}^{\prime}, \ldots, x_{i_{m}}^{\prime}\right) \in[0,1]^{m}$ with $\left\|\left(x_{i_{1}}^{\prime}, \ldots, x_{i_{m}}^{\prime}\right)-\left(x_{i_{1}}, \ldots, x_{i_{m}}\right)\right\|<\eta_{\varepsilon}$ it results $\left\|f\left(x_{i_{1}}^{\prime}, \ldots, x_{i_{m}}^{\prime}\right)-f\left(x_{i_{1}}, \ldots, x_{i_{m}}\right)\right\|<\frac{\varepsilon}{2}$.

Also $\exists M>0$ such that $\left\|f\left(x_{1}, \ldots, x_{m}\right)\right\| \leq M,\left(x_{1}, \ldots, x_{m}\right) \in[0,1]^{m}$.

Let $n_{\varepsilon_{j}} \in \mathbb{N}$, such that:

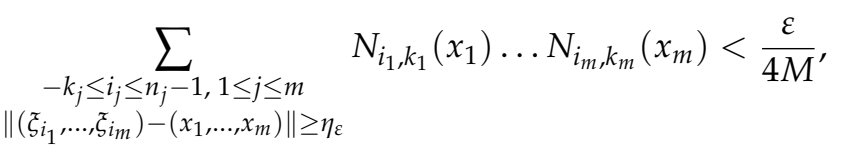

for $n \geq n_{\mathcal{E}_{j}}$. For such $n$ we obtain

$$
\begin{aligned}
& \left|\left(S_{\tilde{\Delta}} f\right)\left(x_{1}, \ldots, x_{m}\right)-f\left(x_{1}, \ldots, x_{m}\right)\right| \\
& \leq \sum_{\begin{array}{c}
-k_{j} \leq i_{j} \leq n_{j}-1,1 \leq j \leq m \\
\left\|\left(\xi_{i_{1}}, \ldots, \xi_{i_{m}}\right)-\left(x_{1}, \ldots, x_{m}\right)\right\| \geq \eta_{\varepsilon}
\end{array}} N_{i_{1}, k_{1}}\left(x_{1}\right) \ldots N_{i_{m}, k_{m}}\left(x_{m}\right)\left|f\left(\xi_{i_{1}}, \ldots, \xi_{i_{m}}\right)-f\left(x_{1}, \ldots, x_{m}\right)\right|
\end{aligned}
$$

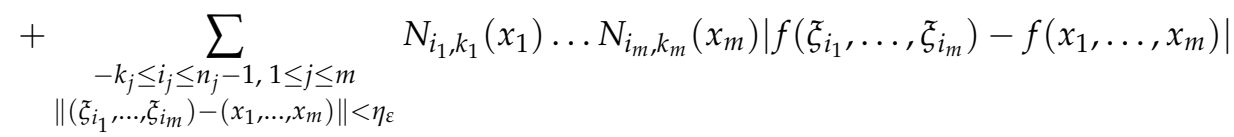

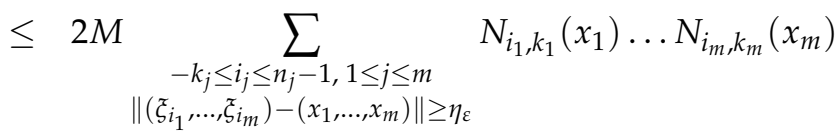

$$
\begin{aligned}
& +\frac{\varepsilon}{2} \sum_{\substack{-k_{j} \leq i_{j} \leq n_{j}-1,1 \leq j \leq m \\
\left\|\left(\xi_{i_{1}}, \ldots, \xi_{i m}\right)-\left(x_{1}, \ldots, x_{m}\right)\right\|<\eta_{\varepsilon}}} N_{i_{1}, k_{1}}\left(x_{1}\right) \ldots N_{i_{m}, k_{m}}\left(x_{m}\right) \\
& \leq 2 M \cdot \frac{\varepsilon}{4 M}+\frac{\varepsilon}{2}=\varepsilon \text {. }
\end{aligned}
$$

The norm of the division $\Delta^{\star}$ is

$$
\left\|\Delta^{\star}\right\|:=\left\|\Delta_{1}\right\|+\left\|\Delta_{2}\right\|+\ldots+\left\|\Delta_{m}\right\|,
$$

where $\left\|\Delta_{j}\right\|=\max _{i_{j}}\left(x_{i_{j+1}}-x_{i_{j}}\right)$.

We use the first order modulus of continuity:

$$
\omega_{1}(f, \rho):=\sup \left\{|f(x)-f(y)|, x, y \in[0,1]^{m},\|x-y\| \leq \rho\right\},
$$

where $f \in C\left([0,1]^{m}\right), \rho>0$.

Theorem 2. For any $f \in C\left([0,1]^{m}\right)$, operators $S_{\Delta^{\star}}$ given in (7) satisfy inequality

$$
\left\|\left(S_{\Delta^{\star}} f\right)-f\right\| \leq \omega_{1}\left(f, \vartheta\left\|\Delta^{\star}\right\|\right),
$$

where $\vartheta=\frac{1}{2} \max _{1 \leq j \leq m}\left\{k_{j}+1\right\}$. 
Proof. Let the continuous function $f$ and $\left(x_{1}, \ldots, x_{m}\right) \in[0,1]^{m}$. For any $1 \leq j \leq m$ there is $q_{j} \in\left\{0,1, \ldots, n_{j}-1\right\}$, such that $x_{j} \in\left[v_{q_{j}}, v_{q_{j}+1}\right]$. Then $N_{i_{j}, k_{j}}\left(x_{j}\right)=0$, for $-k_{j} \leq i_{j}<q_{j}-k_{j}$ and $q_{j}<i_{j} \leq n_{j}-1$.

Let $q_{j}-k_{j} \leq i_{j} \leq q_{j}, 1 \leq j \leq m$. Then

$$
\begin{aligned}
& x_{j}-\xi_{i_{j}} \leq v_{q_{j}+1}-\frac{v_{i_{j}+1}+\ldots+v_{i_{j}+k_{j}}}{k_{j}} \leq v_{q_{j}+1}-\frac{v_{q_{j}-k_{j}+1}+\ldots+v_{q_{j}}}{k_{j}} \\
\leq & \frac{1}{k_{j}}\left(k_{j}+\left(k_{j}-1\right)+\ldots+1\right)\left\|\Delta_{j}\right\|=\frac{k_{j}+1}{2}\left\|\Delta_{j}\right\|
\end{aligned}
$$

and

$$
\begin{aligned}
& x_{j}-\xi_{i_{j}} \geq v_{q_{1}}-\frac{v_{i_{j}+1}+\ldots+v_{i_{j}+k_{j}}}{k_{j}} \geq v_{q_{j}}-\frac{v_{q_{j}+1}+\ldots+v_{q_{j}+k_{j}}}{k_{j}} \\
\geq & -\frac{1}{k_{j}}\left(1+2+\ldots+k_{j}\right)\left\|\Delta_{j}\right\|=-\frac{k_{j}+1}{2}\left\|\Delta_{j}\right\| .
\end{aligned}
$$

Therefore,

$$
\left|x_{j}-\xi_{i_{j}}\right| \leq \frac{k_{j}+1}{2}\left\|\Delta_{j}\right\|, \text { for } q_{j}-k_{j} \leq i_{j} \leq q_{j}, 1 \leq j \leq m .
$$

Then, for $q_{j}-k_{j} \leq i_{j} \leq q_{j}, 1 \leq j \leq m$ we have

$$
\left\|\left(x_{1}, \ldots, x_{m}\right)-\left(\xi_{1}, \ldots, \xi_{m}\right)\right\| \leq \sum_{j=1}^{m}\left|x_{j}-\xi_{i_{j}}\right| \leq \vartheta\left\|\Delta^{\star}\right\| .
$$

It results

$$
\begin{aligned}
& \left|\left(S_{\Delta^{\star}} f\right)\left(x_{1}, \ldots, x_{m}\right)-f\left(x_{1}, \ldots, x_{m}\right)\right| \\
= & \left|\sum_{i_{1}=-k_{1}}^{n_{1}-1} \ldots \sum_{i_{m}=-k_{m}}^{n_{m}-1} N_{i_{1}, k_{1}}\left(x_{1}\right) \ldots N_{i_{m}, k_{m}}\left(x_{m}\right) f\left(\xi_{i_{1}}, \ldots, \xi_{i_{m}}\right)-f\left(x_{1}, \ldots, x_{m}\right)\right| \\
\leq & \sum_{i_{1}=-k_{1}}^{n_{1}-1} \ldots \sum_{i_{m}=-k_{m}}^{n_{m}-1} N_{i_{1}, k_{1}}\left(x_{1}\right) \ldots N_{i_{m}, k_{m}}\left(x_{m}\right)\left|f\left(\xi_{i_{1}}, \ldots, \xi_{i_{m}}\right)-f\left(x_{1}, \ldots, x_{m}\right)\right| \\
= & \sum_{i_{1}=q_{1}-k_{1}}^{q_{j}} \ldots \sum_{i_{m}=q_{m}-k_{m}}^{q_{m}} N_{i_{1}, k_{1}}\left(x_{1}\right) \ldots N_{i_{m}, k_{m}}\left(x_{m}\right)\left|f\left(\xi_{i_{1}}, \ldots, \xi_{i_{m}}\right)-f\left(x_{1}, \ldots, x_{m}\right)\right| \\
\leq & \sum_{i_{1}=q_{1}-k_{1}}^{q_{j}} \ldots \sum_{i_{m}=q_{m}-k_{m}}^{q_{m}} N_{i_{1}, k_{1}}\left(x_{1}\right) \ldots N_{i_{m}, k_{m}}\left(x_{m}\right) \omega_{1}\left(f,\left\|\left(\xi_{i_{1}}, \ldots, \xi_{i_{m}}\right)-\left(x_{1}, \ldots, x_{m}\right)\right\|\right) \\
\leq & \sum_{i_{1}=q_{1}-k_{1}}^{q_{j}} \ldots \sum_{i_{m}=q_{m}-k_{m}}^{q_{m}} N_{i_{1}, k_{1}}\left(x_{1}\right) \ldots N_{i_{m}, k_{m}}\left(x_{m}\right) \omega_{1}\left(f, \vartheta\left\|\Delta^{\star}\right\|\right) \\
= & \omega_{1}\left(f, \vartheta\left\|\Delta^{\star}\right\|\right) .
\end{aligned}
$$

Corollary 1. Multidimensional Schoenberg-type operators

$$
\left(S_{\Delta^{\star}} f\right)\left(x_{1}, \ldots, x_{m}\right)=\sum_{i_{1}=-k_{1}}^{n_{1}-1} \ldots \sum_{i_{m}=-k_{m}}^{n_{m}-1} N_{i_{1}, k_{1}}\left(x_{1}\right) \ldots N_{i_{m}, k_{m}}\left(x_{m}\right) f\left(\xi_{i_{1}}, \ldots, \xi_{i_{m}}\right)
$$

converge uniformly on $[0,1]^{m}$ to $f$, for any continuous function $f$ if $\left\|\Delta^{\star}\right\| \rightarrow 0$. 


\section{Preservation of Monotonicity and Convexity by Multidimensional Schoenberg-Type Operators with Equidistant Knots}

In this section, we will extend some results obtained by Marsden in the case of onedimensional Schoenberg operators.

Let $k \in \mathbb{N}$. We denote by $\left(S_{\Delta} \varphi\right)(x)$ the one-dimensional Schoenberg operators of degree $k$ associated with the knot sequence $\Delta=\left\{v_{i}\right\}_{-k}^{n+k}$, where $v_{-k}=\ldots=v_{0}=0<$ $v_{1}<\ldots<v_{n-1}<1=v_{n}=\ldots=v_{n+k}$, and the Greville abscissas $\xi_{j}=\frac{v_{j+1}+\ldots+v_{j+k}}{k}$, $(\varphi:[0,1] \rightarrow \mathbb{R}, x \in[0,1])$. The B-spline of degree $k$ associated to $\Delta$ is denoted by $N_{j}(x)$, $-k \leq j \leq n-1$. Next, denote $\Delta^{-}=\left\{v_{i}\right\}_{-k+1}^{n+k-1}$. The corresponding B-splines of degree $k-1$ associated with the knot sequence $\Delta^{-}$by $N_{j}^{-}(x), 1-k \leq j \leq n-1$ and the corresponding Greville abscissas is denoted by $\xi_{j}^{-}$. In addition, for $k \geq 2$, denote $\Delta^{=}=\left\{v_{i}\right\}_{-k+2}^{n+k-2}$ and the corresponding B-splines of degree $k-2$ associated with the knot sequence $\Delta^{=}$by $N_{j}^{=}(x)$, $2-k \leq j \leq n-1$. Using these notations, the following relations are given in [3]:

$$
\begin{gathered}
D\left(S_{\Delta} \varphi\right)(x)=\sum_{j=1-k}^{n-1} \frac{f\left(\xi_{j}\right)-f\left(\xi_{j-1}\right)}{\xi_{j}-\xi_{j-1}} N_{j}^{-}(x), \\
D^{2}\left(S_{\Delta} \varphi\right)(x)=\sum_{j=2-k}^{n-1} D^{2} \varphi\left(\eta_{j}\right) \frac{\xi_{j}-\xi_{j-2}}{2\left(\xi_{j}^{-}-\xi_{j-1}^{-}\right)} N_{j}^{=}(x), \text { where } \xi_{j-2}<\eta_{j}<\xi_{j} .
\end{gathered}
$$

In the following theorems it is considered that $n$ and $k$ are variable.

Theorem 3 ([3]). Let $\varphi \in C^{1}[0,1]$ and $\frac{\|\Delta\|}{k} \rightarrow 0$, $\liminf k>1$. Then:

(i) $\left(S_{\Delta} \varphi\right)(x) \rightarrow \varphi(x)$ uniformly on $[0,1]$;

(ii) $D\left(S_{\Delta} \varphi\right)(x) \rightarrow D \varphi(x)$ uniformly on $[0,1]$.

Theorem 4 ([3]). Let $\varphi \in C^{2}[0,1]$ and $x_{i}=\frac{i}{n}, 0<i<n$ the interior knots of $\Delta$. Let $n+k \rightarrow \infty, \lim \inf n>1, \liminf k>1$. Then $\lim D^{2}\left(S_{\Delta} \varphi\right)(x)=D^{2} \varphi(x), 0<x<1$. The convergence is uniform on compact subsets of $(0,1)$.

Theorem 5 ([3]). Let $\varphi \in C^{2}[0,1]$ and $k>2$. Then:

(i) If $D \varphi(x) \geq 0$ on $[0,1]$ then $D\left(S_{\Delta} \varphi\right)(x) \geq 0$ on $[0,1]$;

(ii) If $D^{2} \varphi(x) \geq 0$ on $[0,1]$ then $D^{2}\left(S_{\Delta} \varphi\right)(x) \geq 0$ on $[0,1]$.

We are interested in generalizing these above results in the case of multidimensional Schoenberg-type operators.

Let an integer $m \geq 1$. Denote $D=[0,1]^{m}$.

We consider now multidimensional Schoenberg-type operators with equidistant knots on $D$ of the form

$$
\left(S_{n, k}^{m} f\right)(x)=\sum_{i_{1}, \ldots, i_{m}=-k}^{n-1} N_{i_{1}}\left(x_{1}\right) \ldots N_{i_{m}}\left(x_{m}\right) f\left(\xi_{i_{1}}, \ldots, \xi_{i_{m}}\right),
$$

where $f: D \rightarrow \mathbb{R}, x=\left(x_{1}, \ldots, x_{m}\right) \in D, \xi_{i}^{j}=\frac{v_{i+1}+\ldots+v_{i+k}}{k}$ and

$$
0=v_{-k}=\ldots=v_{0}<v_{1}=\frac{1}{n}<v_{2}=\frac{2}{n}<\ldots<v_{n-1}=\frac{n-1}{n}<v_{n}=\ldots v_{n+k}=1 .
$$

On $D$ consider the following partial order. If $a=\left(a_{1}, \ldots, a_{m}\right) \in D, b=\left(b_{1}, \ldots, b_{m}\right) \in$ $D$, we write $a \leq b$, iff $a_{i} \leq b_{i}$, for $1 \leq i \leq m$. A function $f: D \rightarrow \mathbb{R}$ is said to be increasing if for any $a, b \in D$, such that $a \leq b$, we have $f(a) \leq f(b)$. 
Theorem 6. For any integers $m \geq 1$, and $k \geq 1$, if $f: D \rightarrow \mathbb{R}$ is increasing then $S_{n, k}^{m} f$ is increasing on $D$, for any $n \geq 1$.

Proof. Show that

$$
\left(S_{n, k}^{m} f\right)(a) \leq\left(S_{n, k}^{m} f\right)(b), a, b \in D, a \leq b .
$$

Let $a, b \in D, a \leq b, a \neq b$. Let $v=b-a$. Write $v=\left(v_{1}, \ldots, v_{m}\right)$. Then $v_{i} \geq 0, i=1, m$. In order to show (17) it suffices to show that function $g(t)=\left(S_{n, k}^{m} f\right)(a+t v), t \in[0,1]$, is increasing, and for this it suffices to have $\frac{\mathrm{d}}{\mathrm{d} t}\left(S_{n, k}^{m} f\right)(a+t v) \geq 0$, for $t \in[0,1]$.

We have

$$
\frac{\mathrm{d}}{\mathrm{d} t}\left(S_{n, k}^{m} f\right)(a+t v)=\sum_{j=1}^{m} \frac{\partial}{\partial x_{j}}\left(S_{n, k}^{m} f\right)(a+t v) v_{j} .
$$

Because $v_{i} \geq 0,1 \leq i \leq m$ it suffices to show:

$$
\frac{\partial}{\partial x_{j}}\left(S_{n, k}^{m} f\right)(a+t v) \geq 0,1 \leq j \leq m .
$$

Denoting $x=a+t v, x=\left(x_{1}, \ldots, x_{m}\right)$ one obtains

$$
\begin{aligned}
& \frac{\partial}{\partial x_{j}}\left(S_{n, k}^{m} f\right)(x)=\frac{\partial}{\partial x_{j}} \sum_{i_{1}, \ldots, i_{m}=-k}^{n-1} N_{i_{1}}\left(x_{1}\right) \cdot \ldots \cdot N_{i_{m}}\left(x_{m}\right) f\left(\xi_{i_{1}}, \ldots, x_{i_{m}}\right) \\
= & \sum_{-k \leq i_{1}, \ldots, i_{j-1}, i_{j+1}, \ldots i_{m} \leq n-1} \prod_{l=1, m, l \neq j} N_{i_{l}}\left(x_{l}\right) \frac{\mathrm{d}}{\mathrm{d} x_{j}} \sum_{i_{j}=-k}^{n-1} N_{i_{j}}\left(x_{j}\right) f\left(\xi_{i_{1}}, \ldots, \xi_{i_{m}}\right) .
\end{aligned}
$$

Fix the indices $i_{1}, \ldots, i_{j-1}, i_{j+1}, \ldots i_{m} \in\{-k, \ldots, n-1\}$ and define function $h:[0,1] \rightarrow \mathbb{R}$, given by

$$
h(t)=f\left(\xi_{i_{1}}, \ldots, \xi_{j-1}, t, \xi_{j+1}, \ldots, \xi_{i_{m}}\right), t \in[0,1] .
$$

Using formula (14) we obtain

$\frac{\mathrm{d}}{\mathrm{d} x_{j}} \sum_{i_{j}=-k}^{n-1} N_{i_{j}}\left(x_{j}\right) f\left(\xi_{i_{1}}, \ldots, \xi_{i_{m}}\right)=\frac{\mathrm{d}}{\mathrm{d} x_{j}} \sum_{i_{j}=-k}^{n-1} N_{i_{j}}\left(x_{j}\right) h\left(\xi_{i_{j}}\right)=\sum_{i_{j}=1-k}^{n-1} \frac{h\left(\xi_{i_{j}}\right)-h\left(\xi_{i_{j}-1}\right)}{\xi_{i_{j}}-\xi_{i_{j}-1}} N_{i_{j}}^{-}\left(x_{j}\right)$.

However, $h\left(\xi_{i_{j}}\right)-h\left(\xi_{i_{j}-1}\right) \geq 0$, since $f$ is increasing. In addition, taking into account that $N_{i_{j}}^{-}\left(x_{j}\right) \geq 0,1-k \leq i_{j} \leq n-1$ and $N_{i_{l}}\left(x_{l}\right) \geq 0,-k \leq i_{l} \leq n-1,1 \leq l \leq m, l \neq j$, relation (17) is true.

In the next two theorems we give generalizations of Theorem 4 . We mention that $\stackrel{\circ}{D}$ means the interior of the set $D$.

Theorem 7. Let $k \geq 2$. Let $f: D \rightarrow \mathbb{R}$. For $1 \leq j \leq m$, if $f$ admits the continuous derivatives $\frac{\partial^{2}}{\partial x_{j}^{2}}$ on $D$, then for any compact set $K \subset \stackrel{\circ}{D}$ we have

$$
\lim _{n \rightarrow \infty} \frac{\partial^{2}}{\partial x_{j}^{2}}\left(S_{n, k}^{m} f\right)(x)=\frac{\partial^{2} f}{\partial x_{j}^{2}}(x), \text { uniformly for } x \in K
$$


Proof. It suffices to consider only compacts of the form $K=[a, b]^{m}$, where $[a, b] \subset(0,1)$, because for any compact $K \subset \stackrel{\circ}{D}$ there exists an interval $[a, b] \subset(0,1)$, such that $K \subset[a, b]^{m}$. Using the function $h$ given in (18), we obtain (denoting $i=i_{j}$ ):

$$
\begin{aligned}
\frac{\partial^{2}}{\partial x_{j}^{2}}\left(S_{n, k}^{m} f\right)(x) & =\sum_{i_{1}, \ldots, i_{j-1}, i_{j+1}, \ldots i_{m}=-k}^{n-1} \prod_{l=1, m, l \neq j} N_{l}\left(x_{l}\right) \frac{\mathrm{d}^{2}}{\mathrm{~d} x_{j}^{2}} \sum_{i_{j}=-k}^{n-1} N_{i_{j}}\left(x_{j}\right) f\left(\xi_{i_{1}}, \ldots, \xi_{i_{m}}\right) \\
& =\sum_{-k \leq i_{1}, \ldots, i_{j-1}, i_{j+1}, \ldots i_{m} \leq n-1} \prod_{l=1, m, l \neq j} N_{l}\left(x_{l}\right) \frac{\mathrm{d}^{2}}{\mathrm{~d} x_{j}^{2}} \sum_{i=-k}^{n-1} N_{i}\left(x_{j}\right) h\left(\xi_{i}\right) .
\end{aligned}
$$

Using formula (15) it follows

$$
\frac{\mathrm{d}^{2}}{\mathrm{~d} x_{j}^{2}} \sum_{i=-k}^{n-1} N_{i}\left(x_{j}\right) h\left(\xi_{i}\right)=\sum_{i_{j}=2-k}^{n-1} \frac{\xi_{i}-\xi_{i-2}}{2\left(\xi_{i}^{-}-\xi_{i-1}^{-}\right)} N_{i}^{=}\left(x_{j}\right) D^{2} h\left(\eta_{i}\right)
$$

where $\xi_{i-2}<\eta_{i}<\xi_{i}$.

For $0 \leq i \leq n-k$, we have $\xi_{i}=\xi_{i}^{-}=\frac{v_{i+1}+\ldots+v_{i+k}}{k}=v_{i}+\frac{1+\ldots+k}{k n}=v_{i}+\frac{k+1}{2 n}$. The support of a function $N_{i}$ is the interval $\left[v_{i}, \ldots, v_{i+k+1}\right]$. Then, if $\frac{k+1}{n} \leq a$ and $b+\frac{k+1}{n} \leq 1$, and $x_{j} \in[a, b]$, then $N_{i}^{=}\left(x_{j}\right)=0$, for $-k \leq i \leq-1$, and $n-k+1 \leq i \leq n-1$. In addition, for $2 \leq i \leq n-k$ we obtain $\frac{\xi_{i}-\xi_{i-2}}{2\left(\tilde{\xi}_{i}^{-}-\xi_{i-1}^{-}\right)}=1$, so that we can write more simply

$$
\frac{\mathrm{d}^{2}}{\mathrm{~d} x_{j}^{2}} \sum_{i=-k}^{n-1} N_{i}\left(x_{j}\right) h\left(\xi_{i}\right)=\sum_{i=2}^{n-k} N_{i}^{=}\left(x_{j}\right) D^{2} h\left(\eta_{i}\right), x_{j} \in[a, b] .
$$

Consider the moduli of continuity of functions $D^{2} h$ and $\frac{\partial^{2} f}{\partial x_{j}^{2}}$ :

$$
\begin{aligned}
\omega_{1}\left(D^{2} h, \rho\right) & =\sup \left\{\left|D^{2} h\left(s_{1}\right)-D^{2} h\left(s_{2}\right)\right|, s_{1}, s_{2} \in[0,1],\left|s_{1}-s_{2}\right| \leq \rho\right\} \\
\omega_{1}\left(\frac{\partial^{2} f}{\partial x_{j}^{2}}, \rho\right) & =\sup \left\{\left|\frac{\partial^{2} f}{\partial x_{j}^{2}}\left(y_{1}\right)-\frac{\partial^{2} f}{\partial x_{j}^{2}}\left(y_{2}\right)\right|, y_{1}, y_{2} \in D,\left\|y_{1}-y_{2}\right\| \leq \rho\right\} .
\end{aligned}
$$

where $\rho>0$. Because $D^{2} h$ is a restriction of function $\frac{\partial^{2} f}{\partial x_{j}^{2}}$ we obtain

$$
\omega_{1}\left(D^{2} h, \rho\right) \leq \omega_{1}\left(\frac{\partial^{2} f}{\partial x_{j}^{2}}, \rho\right), \forall \rho>0 .
$$

In addition, since $f$ and has continuous partial second derivatives on $D$, it results that function $\frac{\partial^{2} f}{\partial x_{j}^{2}}$ is uniformly continuous and consequently

$$
\lim _{\rho \rightarrow 0} \omega_{1}\left(\frac{\partial^{2} f}{\partial x_{j}^{2}}, \rho\right)=0 .
$$

We have

$$
\begin{aligned}
& \left|\sum_{i=2}^{n-k} N_{i}^{=}\left(x_{j}\right) D^{2} h\left(\eta_{i}\right)-\sum_{i=2}^{n-k} N_{i}^{=}\left(x_{j}\right) D^{2} h\left(\xi_{i}\right)\right| \leq \sum_{i=2}^{n-k} N_{i}^{=}\left(x_{j}\right) \omega_{1}\left(D^{2} h,\left|\eta_{i}-\xi_{i}\right|\right) \\
\leq & \omega_{1}\left(\frac{\partial^{2} f}{\partial x_{j}^{2}}, \frac{2}{n}\right),
\end{aligned}
$$


since $\sum_{i=2}^{n-k} N_{i}^{=}\left(x_{j}\right)=1$, for $x_{j} \in[a, b]$. It follows

$$
\lim _{n \rightarrow \infty}\left(\sum_{i=2}^{n-k} N_{i}^{=}\left(x_{j}\right) D^{2} h\left(\eta_{i}\right)-\sum_{i=2}^{n-k} N_{i}^{=}\left(x_{j}\right) D^{2} h\left(\xi_{i}\right)\right)=0,
$$

uniformly with regard to indices $i_{1}, \ldots, i_{j-1}, i_{j+1}, \ldots i_{m}$ and $x \in[a, b]^{m}$. On the other hand, consider the sequence of one-dimensional Schoenberg operators $L_{n}: C[0,1] \rightarrow C[0,1]$

$$
\left(L_{n} \varphi\right)(x)=\sum_{i=2}^{n-k} N_{i}^{=}\left(x_{j}\right) \varphi\left(\xi_{i}\right), \varphi \in C[0,1], x_{j} \in[0,1] .
$$

This sequence of positive linear operators approximates uniformly on $[a, b]$ any function $\varphi \in C[0,1]$. Moreover, $L_{n} e_{0}=e_{0}, L e_{1}=e_{1}$. Using the well known estimate of Shisha and Mond, we obtain

$$
\left\|L_{n}\left(D^{2} h\right)-D h^{2}\right\|_{[a, b]} \leq 2 \omega_{1}\left(D^{2} h, \sqrt{\left\|L_{n} e_{2}-e_{2}\right\|}\right) \leq 2 \omega_{1}\left(\frac{\partial^{2} f}{\partial x_{j}^{2}}, \sqrt{\left\|L_{n} e_{2}-e_{2}\right\|}\right) .
$$

Since $\lim _{n \rightarrow \infty}\left\|L_{n} e_{2}-e_{2}\right\|=0$ we get

$$
\lim _{n \rightarrow \infty} \sum_{i=2}^{n-k} N_{i}^{=}\left(x_{j}\right) D^{2} h\left(\eta_{i}\right)=D^{2} h\left(x_{j}\right)
$$

uniformly with regard to $x_{j} \in[a, b]$ and indices $i_{1}, \ldots, i_{j-1}, i_{j+1}, \ldots i_{m}$. From (22) and (23) we deduce

$$
\lim _{n \rightarrow \infty} \sum_{i=2}^{n-k} N_{i}^{=}\left(x_{j}\right) D^{2} h\left(\eta_{i}\right)=\frac{\partial^{2} f}{\partial x_{j}^{2}}\left(\xi_{i_{1}}, \ldots, \xi_{i_{j-1}}, x_{j}, \xi_{i_{j+1}}, \ldots, \xi_{i_{m}}\right),
$$

uniformly with regard to $x \in[a, b]^{m}$ and indices $i_{1}, \ldots, i_{j-1}, i_{j+1}, \ldots i_{m}$. Taking into account relations (20) and (21) we obtain the uniform limit with regard to $x \in[a, b]^{m}$ :

$$
\begin{aligned}
& \lim _{n \rightarrow \infty} \frac{\partial^{2}}{\partial x_{j}^{2}}\left(S_{n, k}^{m} f\right)(x) \\
= & \lim _{n \rightarrow \infty} \sum_{-k \leq i_{1}, \ldots, i_{j-1}, i_{j+1}, \ldots i_{m} \leq n-1} \prod_{l=1, m, l \neq j} N_{l}\left(x_{l}\right) \frac{\partial^{2} f}{\partial x_{j}^{2}}\left(\xi_{i_{1}}, \ldots, \xi_{i_{j-1}}, x_{j}, \xi_{i_{j+1}}, \ldots \xi_{m}\right) .
\end{aligned}
$$

Now consider the $m-1$ - dimensional Schoenberg operator $U_{n}: C\left([0,1]^{m-1}\right) \rightarrow$ $C\left([a, b]^{m-1}\right)$, given by

$$
=\sum_{-k \leq i_{1}, \ldots, i_{j-1}, i_{j+1}, \ldots i_{m} \leq n-1} \prod_{l=1, m, l \neq j} N_{l}\left(x_{l}\right) \varphi\left(\xi_{i_{1}}, \ldots, \xi_{i_{j-1}}, \xi_{i_{j+1}}, \ldots \xi_{m}\right),
$$

where $\varphi \in C\left([0,1]^{m-1}\right)$ and $\left(x_{1}, \ldots, x_{j-1}, x_{j+1}, \ldots, x_{m}\right) \in[a, b]^{m-1}$. With the choice $\varphi\left(u_{1}, \ldots\right.$, $\left.u_{j-1}, u_{j+1}, \ldots, u_{m}\right)=\frac{\partial^{2} f}{\partial x_{j}^{2}}\left(u_{1}, \ldots, u_{j-1}, x_{j}, u_{j+1}, \ldots, u_{m}\right)$, for fixed $x_{j} \in[0,1]$ and $\left(u_{1}, \ldots\right.$, $\left.u_{j-1}, x_{j}, u_{j+1}, \ldots, u_{m}\right) \in[a, b]^{m-1}$ and using Theorem 2 we obtain, for fixed $x_{j}$ :

$$
\left|\left(U_{n} \varphi\right)\left(x_{1}, \ldots, x_{j-1}, x_{j+1}, \ldots, x_{m}\right)-\frac{\partial^{2} f}{\partial x_{j}^{2}}\left(x_{1}, \ldots, x_{m}\right)\right| \leq \omega_{1}\left(\varphi, \frac{k m}{2 n}\right) .
$$




$$
\begin{aligned}
& \text { Since } \omega_{1}\left(\varphi, \frac{k m}{2 n}\right) \leq \omega_{1}\left(\frac{\partial^{2} f}{\partial x_{j}^{2}}, \frac{k m}{2 n}\right) \text { one obtains the uniform majorization with regard to } \\
& \begin{array}{c}
\left(x_{1}, \ldots, x_{m}\right) \in[a, b]^{m}: \\
\mid \prod_{-k \leq i_{1}, \ldots, i_{j-1}, i_{j+1}, \ldots i_{m} \leq n-1} \prod_{l=1, m, l \neq j} N_{l}\left(x_{l}\right) \frac{\partial^{2} f}{\partial x_{j}^{2}}\left(\xi_{i_{1}}, \ldots, \xi_{i_{j-1}}, x_{j}, \xi_{i_{j+1}}, \ldots, \xi_{i_{m}}\right) \\
\quad-\frac{\partial^{2} f}{\partial x_{j}^{2}}\left(x_{1}, \ldots, x_{m}\right) \mid \\
\leq \omega_{1}\left(\frac{\partial^{2} f}{\partial x_{j}^{2}}, \frac{k m}{2 n}\right) .
\end{array}
\end{aligned}
$$

Finally, it results that (19) is true.

Theorem 8. Let $k \geq 2$ and $f: D \rightarrow \mathbb{R}$. For indices $1 \leq j_{1}<j_{2} \leq m$ if $f$ admits the continuous second derivative $\frac{\partial f}{\partial x_{j_{1}} \partial x_{j_{2}}}$ on $D$, then we have

$$
\lim _{n \rightarrow \infty} \frac{\partial^{2}}{\partial x_{j_{1}} \partial x_{j_{2}}}\left(S_{n, k}^{m} f\right)(x)=\frac{\partial^{2} f}{\partial x_{j_{1}} \partial x_{j_{2}}}(x), \text { uniformly for } x \in D \text {. }
$$

Proof. For $x=\left(x_{1}, \ldots, x_{m}\right) \in D$ we find

$$
\begin{gathered}
\frac{\partial^{2}}{\partial x_{j_{1}} \partial x_{j_{2}}}\left(S_{n, k}^{m} f\right)(x)=\sum_{-k \leq i_{l} \leq n-1,1 \leq l \leq m, l \neq j_{1}, j_{2}} \prod_{l=1, m, l \neq j_{1}, j_{2}} N_{l}\left(x_{l}\right) \\
\times \frac{\mathrm{d}}{\mathrm{d} x_{j_{1}}} \sum_{r=-k}^{n-1} N_{r}\left(x_{j_{1}}\right) \frac{\mathrm{d}}{\mathrm{d} x_{j_{2}}} \sum_{s=-k}^{n-1} N_{s}\left(x_{j_{2}}\right) g\left(\xi_{r}, \xi_{s}\right),
\end{gathered}
$$

where we denoted by $g$, the function

$$
g(u, v)=f\left(\xi_{1}, \ldots, \xi_{j_{1}-1}, u, \xi_{j_{1}+1}, \ldots, \xi_{j_{2}-1}, v, \xi_{j_{2}+1}, \ldots, \xi_{m}\right),(u, v) \in[0,1]^{2}
$$

and $r=i_{j_{1}}$ and $s=i_{j_{2}}$. Using formula (14) two times, it follows that we can write

$$
\begin{aligned}
\frac{\mathrm{d}}{\mathrm{d} x_{j_{1}}} \sum_{r=-k}^{n-1} N_{r}\left(x_{j_{1}}\right) & \frac{\mathrm{d}}{\mathrm{d} x_{j_{2}}} \sum_{s=-k}^{n-1} N_{s}\left(x_{j_{2}}\right) g\left(\xi_{r}, \xi_{s}\right)=\sum_{r=-k}^{n-1} \sum_{s=-k}^{n-1} N_{r}^{-}\left(x_{j_{1}}\right) N_{s}^{-}\left(x_{j_{2}}\right) \\
& \times \frac{g\left(\xi_{r}, \xi_{s}\right)-g\left(\xi_{r}, \xi_{s-1}\right)-g\left(\xi_{r-1}, \xi_{s}\right)+g\left(\xi_{r-1}, \xi_{s-1}\right)}{\left(\xi_{r}-\xi_{r-1}\right)\left(\xi_{s}-\xi_{s-1}\right)} .
\end{aligned}
$$

We have the limit

$$
\lim _{n \rightarrow \infty}\left(\frac{g\left(\xi_{r}, \xi_{s}\right)-g\left(\xi_{r}, \xi_{s-1}\right)-g\left(\xi_{r-1}, \xi_{s}\right)+g\left(\xi_{r-1}, \xi_{s-1}\right)}{\left(\xi_{r}-\xi_{r-1}\right)\left(\xi_{s}-\xi_{s-1}\right)}-\frac{\partial^{2}}{\partial u \partial v} g\left(\xi_{r}, \xi_{s}\right)\right)=0,
$$

uniformly with regard to index $\xi_{l}, 1 \leq l \leq m$ and $\left(x_{1}, \ldots, x_{m}\right) \in D$. It follows that the limit

$$
\begin{aligned}
& \lim _{n \rightarrow \infty} \sum_{r=-k}^{n-1} \sum_{s=-k}^{n-1} N_{r}^{-}\left(x_{j_{1}}\right) N_{s}^{-}\left(x_{j_{2}}\right) \frac{g\left(\xi_{r}, \xi_{s}\right)-g\left(\xi_{r}, \xi_{s-1}\right)-g\left(\xi_{r-1}, \xi_{s}\right)+g\left(\xi_{r-1}, \xi_{s-1}\right)}{\left(\xi_{r}-\xi_{r-1}\right)\left(\xi_{s}-\xi_{s-1}\right)} \\
= & \lim _{n \rightarrow \infty} \sum_{r=-k}^{n-1} \sum_{s=-k}^{n-1} N_{r}^{-}\left(x_{j_{1}}\right) N_{s}^{-}\left(x_{j_{2}}\right) \frac{\partial^{2}}{\partial u \partial v} g\left(\xi_{r}, \xi_{s}\right),
\end{aligned}
$$

is uniform with regard to $\xi_{l}, 1 \leq l \leq m$ and $\left(x_{1}, \ldots, x_{m}\right) \in D$. 
Using the property of uniform approximation of two-dimensional Schoenberg operators

$$
\sum_{r=-k}^{n-1} \sum_{s=-k}^{n-1} N_{r}^{-}\left(x_{j_{1}}\right) N_{s}^{-}\left(x_{j_{2}}\right) \varphi\left(\xi_{r}\right), \varphi \in C\left([0,1]^{2}\right),
$$

the Shisha and Mond estimate and the inequality $\omega_{1}(g, \rho) \leq \omega_{1}\left(\frac{\partial^{2} f}{\partial x_{j_{1}} \partial x_{j_{2}}}, \rho\right)$, for $\rho>0$, we get, like in Theorem 7

$$
\lim _{n \rightarrow \infty} \sum_{r=-k}^{n-1} \sum_{s=-k}^{n-1} N_{r}^{-}\left(x_{j_{1}}\right) N_{s}^{-}\left(x_{j_{2}}\right) \frac{\partial^{2}}{\partial u \partial v} g\left(\xi_{r}, \xi_{s}\right)=g\left(x_{j_{1}}, x_{j_{2}}\right)
$$

and this limit is uniform with regard to $x \in D$ and indices $i_{l}, 1 \leq l \leq m, l \neq i_{j_{1}}, i_{j_{2}}$. It follows that

$$
\lim _{n \rightarrow \infty} \frac{\mathrm{d}}{\mathrm{d} x_{j_{1}}} \sum_{r=-k}^{n-1} N_{r}\left(x_{j_{1}}\right) \frac{\mathrm{d}}{\mathrm{d} x_{j_{2}}} \sum_{s=-k}^{n-1} N_{s}\left(x_{j_{2}}\right) g\left(\xi_{r}, \xi_{s}\right)=\frac{\partial^{2}}{\partial u \partial v} g\left(x_{j_{1}}, x_{j_{2}}\right)
$$

and the limit is uniform with regard to $x \in D$ and indices $i_{l}, 1 \leq l \leq m, l \neq i_{j_{1}}, i_{j_{2}}$. Then we have the limit

$$
\begin{aligned}
& \lim _{n \rightarrow \infty} \frac{\partial^{2}}{\partial x_{j_{1}} \partial x_{j_{2}}}\left(S_{n, k}^{m} f\right)(x) \\
= & \lim _{n \rightarrow \infty} \sum_{-k \leq i_{l} \leq n-1,1 \leq l \leq m, l \neq j_{1}, j_{2} l=1, m, l \neq j_{1}, j_{2}} N_{l}\left(x_{l}\right) \frac{\partial^{2}}{\partial u \partial v} g\left(x_{j_{1}}, x_{j_{2}}\right) \\
= & \lim _{n \rightarrow \infty} \sum_{-k \leq i_{l} \leq n-1,1 \leq l \leq m, l \neq j_{1}, j_{2}} \prod_{l=1, m, l \neq j_{1}, j_{2}} N_{l}\left(x_{l}\right) \\
& \quad \times \frac{\partial^{2}}{\partial x_{j_{1}} \partial x_{j_{2}}} f\left(\xi_{1}, \ldots, \xi_{j_{1}-1}, x_{j_{1}}, \xi_{j_{1}+1}, \ldots, \xi_{j_{2}-1}, x_{j_{2}}, \xi_{j_{2}+1}, \ldots, \xi_{m}\right),
\end{aligned}
$$

uniform with regard to $x \in D$. Finally, we apply the property of uniform approximation of the Schoenberg operators of degree $m-2$ :

$$
\sum_{-k \leq i_{l} \leq n-1,1 \leq l \leq m, l \neq j_{1}, j_{2}} \prod_{l=1, m, l \neq j_{1}, j_{2}} N_{l}\left(x_{l}\right) \psi\left(\xi_{1}, \ldots, \xi_{j_{1}-1}, \xi_{j_{1}+1}, \ldots, \xi_{j_{2}-1}, \xi_{j_{2}+1}, \ldots, \xi_{m}\right),
$$

to the function

$$
\begin{aligned}
& \psi\left(u_{1}, \ldots, u_{j_{1}-1}, u_{j_{1}+1}, \ldots, u_{j_{2}-1}, u_{j_{2}+1}, \ldots, u_{m}\right) \\
& =\frac{\partial^{2}}{\partial x_{j_{1}} \partial x_{j_{2}}} f\left(u_{1}, \ldots, u_{j_{1}-1}, x_{j_{1}}, u_{j_{1}+1}, \ldots, u_{j_{2}-1}, x_{j_{2}}, u_{j_{2}+1}, \ldots, u_{m}\right),
\end{aligned}
$$

with $x_{j_{1}}$ and $x_{j_{2}}$ fixed. One obtains relation (24).

Theorem 9. If $f: D \rightarrow \mathbb{R}$ is strictly convex and has continuous partial second derivatives on $D$, then for any compact convex set $K \subset \stackrel{\circ}{D}$ there exists an indice $n_{0}$, depending on $f$ and $K$, such that $S_{n, k}^{m}(f)$ is convex for each $n \geq n_{0}$ on $K$.

Proof. From the hypothesis we obtain the following symmetric positive definite quadratic form:

$$
F(x, v):=\sum_{i, j=1}^{n} \frac{\partial^{2} f(x)}{\partial x_{i} \partial x_{j}} v_{i} v_{j}>0, \forall x \in D, \forall v=\left(v_{1}, \ldots, v_{m}\right) \neq 0 .
$$

Denote $B=\left\{x \in \mathbb{R}^{m},\|x\|=1\right\}$. Because $D$ is compact and $B$ is compact we obtain that $D \times B$ is compact. Because the function $F: D \times B \rightarrow \mathbb{R}$ is continuous and strictly 
positive on the domain of definition, from the Weierstrass theorem one obtains that there exists $\mu>0$ such that

$$
\sum_{i, j=1}^{n} \frac{\partial^{2} f(x)}{\partial x_{i} \partial x_{j}} v_{i} v_{j} \geq \mu, \forall x \in D, \forall v \in B
$$

Using Theorems 7 and 8 we obtain

$$
\lim _{n \rightarrow \infty} \frac{\partial^{2}}{\partial x_{i} \partial x_{j}}\left(S_{n, k}^{m} f\right)(x)=\frac{\partial^{2} f(x)}{\partial x_{i} \partial x_{j}} f(x), \text { uniformly for } x \in K,
$$

for any indices $1 \leq i, j \leq m$.

Then it results

$$
\lim _{n \rightarrow \infty} \sum_{i, j=1}^{n} \frac{\partial^{2}}{\partial x_{i} \partial x_{j}}\left(S_{n, k}^{m} f\right)(x) v_{i} v_{j}=\sum_{i, j=1}^{n} \frac{\partial^{2} f(x)}{\partial x_{i} \partial x_{j}} v_{i} v_{j},
$$

uniformly for $x \in K$ and for $v \in B$. Therefore, there exists $n_{0} \in \mathbb{N}$, such that

$$
\sum_{i, j=1}^{n} \frac{\partial^{2}}{\partial x_{i} \partial x_{j}}\left(S_{n, k}^{m} f\right)(x) v_{i} v_{j} \geq \frac{\mu}{2}, x \in K, v \in B
$$

Inequality (27) says that $S_{n, k}^{m}(f)$ is convex on $K$.

\section{Multidimensional Schoenberg-Type Operators of Degree Three on Equidistant Knots}

Let one consider the case with $k_{j}=3 ; n_{j}=n$; the equidistant knots $v_{i_{j}}=\frac{i_{j}}{n}, 0 \leq i_{j} \leq n$, where $1 \leq j \leq m$; the extra-knots $v_{i_{-3}}=v_{i_{-2}}=v_{i_{-1}}=0$ and $v_{i_{n+1}}=v_{i_{n+2}}=v_{i_{n+3}}=1$.

The Greville abscissas are

$$
\xi_{i_{j}}:=\frac{v_{i_{j}+1}+v_{i_{j}+2}+v_{i_{j}+3}}{3}=\left\{\begin{array}{cc}
v_{i_{j}+2}, & i_{j} \in\{-3, \ldots, n-1\} \backslash\{-2, n-2\} \\
\frac{1}{3 n}, & i_{j}=-2, \\
\frac{3 n-1}{3 n} & i_{j}=n-2
\end{array}\right.
$$

with $1 \leq j \leq m$.

The B-splines are

$$
N_{i_{j}, 3}\left(x_{j}\right)=\left(v_{i_{j}+4}-v_{i_{j}}\right)\left[v_{i_{j}}, v_{i_{j}+1}, v_{i_{j}+2}, v_{i_{j}+3}, v_{i_{j}+4}\right]\left(\cdot-x_{j}\right)_{+}^{3},
$$

with $1 \leq j \leq m$.

Multidimensional Schoenberg-type operators with equidistant knots, denoted in the sequel by $S_{n, 3}^{m}$, for $k_{j}=3, n_{j}=n, 1 \leq j \leq m$, are:

$$
\left(S_{n, 3}^{m} f\right)\left(x_{1}, \ldots, x_{m}\right)=\sum_{i_{1}=-3}^{n_{1}-1} \ldots \sum_{i_{m}=-3}^{n_{m}-1} N_{i_{1}, 3}\left(x_{1}\right) \ldots N_{i_{m}, 3}\left(x_{m}\right) f\left(\xi_{i_{1}}, \ldots, \xi_{i_{m}}\right) .
$$

In this section we present certain special results for the cubic splines, which can be proved analogously as in [11].

Lemma 1. The second moment of the multidimensional Schoenberg-type operators $S_{n, 3}^{m}$, with $n \geq 5$ and $\left(x_{1}, \ldots, x_{m}\right) \in[0,1]^{m}$, verifies the relations

$$
\left(S_{n, 3}^{m}\left(\sum_{j=0}^{m}\left(\pi_{j}-x_{j} e_{0}\right)^{2}\right)\right)\left(x_{1}, \ldots, x_{m}\right) \leq \frac{m}{3 n^{2}} .
$$


Moreover,

$$
\left(S_{n, 3}^{m}\left(\sum_{j=0}^{m}\left(\pi_{j}-x_{j} e_{0}\right)^{2}\right)\right)\left(x_{1}, \ldots, x_{m}\right)=\frac{m}{3 n^{2}}
$$

for $x_{j} \in\left[\frac{2}{n}, \frac{n-2}{n}\right]$ and $1 \leq j \leq m$.

Using Lemma and the inequality given in [21]:

$$
\left(S_{n, k}\left(e_{1}-x_{j} e_{0}\right)^{4}\right)\left(x_{j}\right) \leq\left(\frac{k+1}{2 n}\right)^{2}\left(S_{n, k}\left(e_{1}-x_{j} e_{0}\right)^{2}\right)\left(x_{j}\right),
$$

where $S_{n, k}$ denotes the Schoenberg one-dimensional operator of order $k$ with equidistant knots, one obtains:

Lemma 2. For $n \geq 5$ we have

$$
\left(S_{n, 3}^{m}\left(\sum_{j=1}^{m}\left(\pi_{j}-x_{j} e_{0}\right)^{2}\right)\right)\left(x_{1}, \ldots, x_{m}\right) \leq \frac{4 m}{3 n^{4}} .
$$

From Lemma 1 and Lemma 2 and the fact that Schoenberg preserves linear functions, one can deduce the following Voronovskaja-type result, in a similar mode as in [11].

Theorem 10. The following limit is true:

$$
\lim _{n \rightarrow \infty} n^{2}\left(\left(S_{n, 3}^{m} f\right)\left(x_{1}, \ldots, x_{m}\right)-f\left(x_{1}, \ldots, x_{m}\right)\right)=\frac{1}{6} \sum_{j=1}^{m} \frac{\partial^{2} f}{\partial x_{j}^{2}}\left(x_{1}, \ldots, x_{m}\right),
$$

for any $f \in C^{2}\left([0,1]^{m}\right),\left(x_{1}, \ldots, x_{m}\right) \in(0,1)^{m}$

Because Schoenberg preserves linear functions there exists the possibility of expressing the degree of approximation in a more refined mode, using second order moduli of continuity. The following estimates can be obtained similarly to [11] by applying certain general estimates with moduli of continuity proved in [19].

Firstly, consider the usual second order modulus

$$
\tilde{\omega}_{2}(f, h):=\sup \left\{\left|f(u)-2 f\left(\frac{u+v}{2}\right)+f(v)\right|, u, v \in D,\|u-v\| \leq 2 h\right\}
$$

where $f \in C(D), h>0$

One obtains:

\section{Theorem 11.}

$$
\left|\left(S_{n, 3}^{m} f\right)\left(x_{1}, \ldots, x_{m}\right)-f\left(x_{1}, \ldots, x_{m}\right)\right| \leq m\left(1+\frac{1}{6 h^{2} n^{2}}\right) \tilde{\omega}_{2}(f, h),
$$

where $f \in C\left([0,1]^{m}\right), h>0,\left(x_{1}, \ldots, x_{m}\right) \in[0,1]^{m}, n \in \mathbb{N}, n \geq 5$.

Consequently:

$$
\left\|\left(S_{n, 3}^{m} f\right)-f\right\| \leq \frac{7 m}{6} \tilde{\omega}_{2}\left(f, \frac{1}{n}\right), f \in C\left([0,1]^{m}\right), n \in \mathbb{N}, n \geq 5 .
$$

A global second modulus of continuity can be defined by: 


$$
\begin{aligned}
\tilde{\omega}_{2}^{\star}(f, \rho)= & \sup \left\{\left|\sum_{i=1}^{n} \lambda_{i} f\left(\alpha_{i}\right)-f(\alpha)\right|, \alpha \in \mathcal{A}, \alpha_{i} \in D,\left\|\alpha_{i}-\alpha\right\| \leq h,\right. \\
& \left.\lambda_{i} \in(0,1), 0 \leq i \leq n, \lambda_{1}+\ldots+\lambda_{n}=1\right\} .
\end{aligned}
$$

Using this modulus one can obtain an estimate which is independent on the dimension $m$ :

Theorem 12. Let the function $f$ continue on $[0,1]^{m}$ and $h>0$. We have

$$
\left|\left(S_{n, 3}^{m} f\right)\left(x_{1}, \ldots, x_{m}\right)-f\left(x_{1}, \ldots, x_{m}\right)\right| \leq\left(1+\frac{m}{3 n^{2} h^{2}}\right) \tilde{\omega}_{2}^{\star}(f, h) .
$$

Consequently,

$$
\left\|\left(S_{n, 3}^{m} f\right)-f\right\| \leq\left(1+\frac{m}{3}\right) \tilde{\omega}_{2}^{\star}\left(f, \frac{1}{n}\right), f \in C\left([0,1]^{m}\right), n \in \mathbb{N} .
$$

Remark 2. The usual polynomial operators used in approximation have an approximation order only of the type $O\left(\omega_{2}\left(f, \frac{1}{\sqrt{n}}\right)\right), f \in C[0,1]$.

\section{Conclusions}

The Schoenberg operators are practical tools to approximate functions, knowing the values of them in a finite number of points. Schoenberg operators attach to a function a particular type of spline of a freely chosen degree. It is not necessary to use high-degree splines in order to obtain a desired approximation order. It is usually sufficient to use $3 \mathrm{rd}$ order splines. This makes the calculation volume substantially lower than in the case of polynomial approximation.

In approximation of functions, the degree of approximation is not the unique objective. The preservation of certain shape properties of functions is also worth studying. Among these supplementary preservation proprieties, two special types are usually studied: the possibility of simultaneous approximation of functions and of their derivatives of different orders and the preservation of convexity of different orders, including the monotonicity and the usual convexity. These types of properties are known to be true for the one-dimensional case of Schoenberg operators. We put in evidence that they are true in great measure for the multidimensional case. It is well known also that maybe the more important polynomial approximation operators, namely the Bernstein operators, have very good properties for preserving different behaviors of functions. In fact, it is natural that the Schoenberg operators, which can be regarded as generalizations of Bernstein operators, maintain at least in part these good properties. On the other hand, by taking into account that Schoenberg operators offer a great improvement of the order of approximation for the same order of computation, they turn out to be a very powerful tool in the theory of function approximation. In this direction, other properties of preserving certain classes of functions or the simultaneous approximation can be taken into account for further studies.

The results obtained in this paper are connected to the notion of symmetry in several aspects, namely, in the construction of operators, in using the symmetric tools in estimates and in property of convexity, which is given using symmetrical expressions. We believe that this paper can offer a useful tool to specialists with concerns in many areas of practical approximation. 
Author Contributions: R.P. and C.L.M. prepared, wrote, edited and reviewed the paper. Both authors have read and agreed to the published version of the manuscript.

Funding: This research received no external funding.

Institutional Review Board Statement: Not applicable.

Informed Consent Statement: Not applicable.

Data Availability Statement: Not applicable.

Conflicts of Interest: The authors declare no conflict of interest.

\section{References}

1. Schumaker, L.L. Spline Functions: Basic Theory, 3rd ed.; Cambridge University Press: Cambridge, UK, 2007.

2. Schoenberg, I.J. On variation diminishing approximation methods. In On Numerical Approximation; Langer, R.E., Ed.; University of Wisconsin Press: Madison, WI, USA, 1959; pp. 249-274.

3. Marsden, M.J. An identity for spline functions with applications to variation diminishing spline approximation. J. Approx. Theory 1970, 3, 7-49. [CrossRef]

4. Gonska, H.; Kacsó, D.; Tachev, G. Direct estimates and Bernstein-type inequalities for Schoenberg splines. In Mathematical Analysis and Approximation Theory, the 5th Romanian-German Seminar on Approximation Theory and Its Applications; Lupaş, A., Gonska, H., Lupaş. L., Eds.; Burg Verlag: Sibiu, Romania, 2002; pp. 1-10.

5. Beutel, L.; Gonska, H.; Kacsó, D.; Tachev, G. On variation-diminishing Schoenberg operators: New quantitative statements. Monogr. Acad. Cienc. Zaragoza 2002, 20, 9-58.

6. Beutel, L.; Gonska, H.; Kacsó, D.; Tachev, G. Variational-diminishing splines revisited. In Proceedings of the International Symposium on Numerical Analysis and Approximation Theory, Cluj-Napoca, Romania, 9-11 May 2002; Trîmbiţaş, R.T., Ed.; Cluj Napoca University Press: Cluj-Napoca, Romania, 2002; pp. 54-75.

7. Beutel, L.; Gonska, H.; Kacso, D.; Tachev, G. On the second moments of variation-diminishing splines. J. Concr. Appl. Anal. 2004, 2, 91-117.

8. Tachev, G. A lower bound for the second moment of Schoenberg operator. Gen. Math. 2008, 16, 165-170.

9. Beutel, L. On a lower bound of the second moment of the quadratic Schoenberg operator. In Mathematical Analysis and Approximation Theory, the 5th Romanian-German Seminar on Approximation Theory and Its Applications; Lupaş, A., Gonska, H., Lupaş. L., Eds.; Burg Verlag: Sibiu, Romania, 2002; pp. 27-44.

10. Moldovan, C.L.; Păltănea, R. Second degree Schoenberg operators with knots at the roots of Chebyshev polynomials. Rev. Real Acad. Cienc. Exactas Fis. Nat. Ser. A Mat. 2019, 113, 2793-2804. [CrossRef]

11. Moldovan C.L.; Păltănea R. The Exact Form of the Second Moment of Third Degree Schoenberg Spline Operators. Numer. Funct. Anal. Optim. 2020, 41, 1308-1325. [CrossRef]

12. Moldovan C.L.; Păltănea R. A Definition of Two-Dimensional Schoenberg Type Operators. Symmetry 2020, 12, 1364. [CrossRef]

13. Dahmen, W. Approximation by Linear Combinations of Multivariate B-splines. J. Approx. Theory 1981, 31, 299-324. [CrossRef]

14. Chui, K.C. Multivariate Splines; Society for Industrial and Applied Mathematics: Philadelphia, PA, USA, 1988.

15. Sauer, T. Computational aspects of multivariate polynomial interpolation. Adv. Comput. Math. 1995, 3, 219-237. [CrossRef]

16. Bozzini, M.; Lenarduzzi, L.; Rossini, M. Kernel B-splines on general lattices. J. Comput. Appl. Math. 2010, 233, 1620-1630. [CrossRef]

17. Ranirina, D.; Villiers, J. On Hermite vector splines and multi-wavelets. J. Comput. Appl. Math. 2019, 349, 366-378. [CrossRef]

18. Kreuzer, W. Using B-spline frames to represent solutions of acoustics scattering problems. J. Comput. Appl. Math. 2019, 351, 331-343. [CrossRef]

19. Păltănea, R. Approximation Theory Using Positive Linear Operators; Birhäuser: Boston, MA, USA, 2004.

20. Moldovan, C.L.; Păltănea, R.; Visa, I. Improvement of clear sky models for direct solar irradiance considering turbidity factor variable during the day. Renew. Energy 2020, 161, 559-569. [CrossRef]

21. Tachev, G. Voronovskaja's theorem for Schoenberg operator. Math. Inequal. Appl. 2012, 15, 49-59. [CrossRef] 